\title{
Radio Resource Allocator for OFDMA Wireless Systems Based On Genetic Algorithm
}

\author{
Francisco Rafael M. Lima*, Raimundo A. de Oliveira Neto*, Ricardo B. dos Santos*, Walter C. Freitas Jr. and \\ Francisco Rodrigo P. Cavalcanti
}

\begin{abstract}
In this article we formulate a new Radio Resource Allocation problem in Orthogonal Frequency Division Multiple Access systems. The formulated problem has the objective of maximizing the number of User Equipments with fulfilled rate requirements. In order to solve the conceived problem, we use Genetic Algorithms in its modeling. The gains of the solution to this problem are shown in simulation results.
\end{abstract}

Keywords-OFDMA, Genetic Algorithm, RRA and user satisfaction.

Resumo-Neste artigo nós formulamos um novo problema de Alocação de Recursos de Rádio em sistemas de Acesso Múltiplo por Divisão de Frequência Ortogonal. O problema formulado tem o objetivo de maximizar o número de usuários satisfeitos com seus requerimentos de taxa. Para resolver este problema, nós usamos Algoritmos Genéticos em sua modelagem. Os ganhos da solução deste problema são mostrados por meios de resultados de simulação computacional.

Palavras-Chave-OFDMA, Algoritmo Genético, RRA e satisfação do usuário.

\section{INTRODUCTION}

The main standard bodies are planning to use Orthogonal Frequency Division Multiple Access (OFDMA) as the multiple access scheme of future wireless networks such as Long Term Evolution (LTE) and Worldwide Interoperability for Microwave Access (WiMAX). The reasons for this are several. Firstly, these schemes can combat multipath fading problems caused by multiple copies of the transmitted signal which arrives at the receiver with different time delays and attenuations by using narrowband subcarriers that experience approximately flat fading channels. Secondly, because of the smaller frequency spacing between subcarriers allowed by the orthogonality, these schemes have a high spectral efficiency. Finally, OFDMA systems are scalable to high data rates and offer many possibilities of which Radio Resource Allocation (RRA) algorithms take advantage.

OFDMA systems offer a new dimension in diversity to RRA algorithms: the frequency diversity. Due to channel selectivity, it is likely that a given User Equipment (UE) can have some subcarriers in bad and good channel state simultaneously. Therefore, the resource allocator can increase the system efficiency even more by assigning only the good subcarriers

\footnotetext{
* These students are supported by a scholarship granted by FUNCAP. This work was supported by a grant from Ericsson of Brazil - research branch under ERBB/UFC.18 technical cooperation contract. The authors are from Wireless Telecommunication Research Group - GTEL / Department of Teleinformatics - DETI / Federal University of Ceará - UFC. Emails: \{neto,rafaelm,brauner,walter,rodrigo\}@gtel.ufc.br
}

to this UE. Besides, in point-to-multipoint connections, opportunistic RRA can benefit from the multi-user diversity.

Many RRA problems have been formulated in the literature in order to fulfill the UE requirements while utilizing the OFDMA system resources efficiently in downlink [1]. The Rate Maximization (RM) problem intends to allocate resources in such a way as to maximize the system spectral efficiency. The RM problem has a simple solution which consists in allocating the subcarriers to the UEs in better channel state in each subcarrier and performing water filling algorithms to distribute power among subcarriers. However the solution to this problem can cause starvation of UEs with poor channel conditions. The Margin Adaptive (MA) problem has the objective of minimizing the total used power constrained to UE rate requirements subjected to a constant power budget. Another problem is the Rate Adaptive (RA) problem which aims at maximizing the minimum UE data rate. These two other problems are claimed to be NP-complete problems and no efficient optimal solution are known [1].

Combinatorial problems like MA and RA cannot be solved in a optimal way analytically. One of the options is the brute force enumeration, but this method is impractical. Other works have aimed at solving these problems in a suboptimal way [2], [3]. One interesting option is the use of heuristic algorithms like the Genetic Algorithm (GA). The employment of evolutionary algorithms in the telecommunications branch has risen since 1992. Some of the earliest evolutionary telecommunications papers focused on areas like network dimensioning, routing, call admission and frequency assignment (see e.g. [4] for a survey). Nowadays, the evolutionary concepts and biological-inspired approaches are applied in most of telecommunications areas. Features provided by GAs, such as search diversity, effectiveness and robustness, are important for combinatorial optimization problems as those found in OFDMA. GAs have proved to be effective because of their ability to exploit favorable characteristics of previous attempts to construct better solutions. The robustness lies in their power to deal with discontinuities and large multi-modal noisy search spaces [5]. GA has been utilized to solve problems in OFDMA systems in [6] where it considers the problem of total power minimization needed for an Orthogonal Frequency Division Multiplexing (OFDM) symbol, subject to Quality of Service (QoS) restrictions. The QoS restrictions refer to a maximum target Bit Error Rate (BER) and the number of bits of each UE that need to be transmitted in an OFDM symbol.

In our paper, we propose a new RRA problem in the 
OFDMA context so that the number of UEs with fulfilled bit rate requirements are maximized. Furthermore, we provide the modeling to solve the problem utilizing GA although other heuristic tools could be used for the same problem. The remainder of the paper is organized as follows. Section II presents the system modeling. Section III shows the problem formulation and the modeling utilized to solve the problem with GA, and also describes the algorithms that are used for comparison. Finally in sections IV and V, the numerical results achieved by simulation and the conclusions are provided, respectively.

\section{SyStem MODELING}

In the modeled OFDMA system, in order to overcome the frequency selective fading, the bandwidth of each subcarrier is chosen to be sufficiently smaller than the coherence bandwidth of the channel. Therefore, subcarriers are modeled as $N$ flat Rayleigh fading channels correlated in time and frequency.

The subcarriers are shared by $J$ UEs using a Non-real time (NRT) service characterized by a minimum rate requirement $R_{\text {req }}$ which has to be fulfilled at each Transmission Time Interval (TTI). It is assumed that the UEs have always a full data buffer. In addition, a fluid model is assumed for the traffic, i.e., the UE data is completely divisible. The base station is assumed to have knowledge of the channel gain $h_{j, n}$ of each UE $j$ in each subcarrier $n$.

The Signal-to-Noise Ratio (SNR) $\gamma_{j, n}$ of UE $j$ in subcarrier $n$ is defined as

$$
\gamma_{j, n}=\frac{h_{j, n} \cdot p}{\sigma^{2}}
$$

where $p$ is the subcarrier transmit power, that is considered equal in all subcarriers, and $\sigma^{2}$ is the Additive White Gaussian Noise (AWGN) noise power.

The transmit data rate $\mathcal{R}_{j, n}$ in each subcarrier is given by

$$
\mathcal{R}_{j, n}=w \cdot \log _{2}\left(1+\frac{\gamma_{j, n}}{\Gamma}\right)
$$

where $w$ is the subcarrier bandwidth and

$$
\Gamma=-\frac{\ln (5 \cdot B E R)}{1.5},
$$

is the SNR gap for the system BER requirements [7].

To represent the finite Modulation and Coding Schemes (MCSs) of the system, the capacity curve was sampled in the M-Quadrature Amplitude Modulation (QAM) modulations ( $M=2^{m} ; m=1,2,3,4,5$ and 6$)$ as can be seen in Fig. 1. Therefore, the set of possible rates in each subcarrier is not any more continuous and has only 6 values $\mathcal{R}_{j, n}^{m}$, one for each MCS level $m$.

The allocated data rate for each UE $j$ is designed by $r_{j}$ and depends on the assignment vector $\mathbf{x}$ whose integer elements $x_{n}$ $(n=1,2, \ldots, N)$ represent the UE assigned to the subcarrier $n$.

Once the subcarrier and bit allocation are defined by the scheduler, it is assumed that this information is sent via a separate control channel.

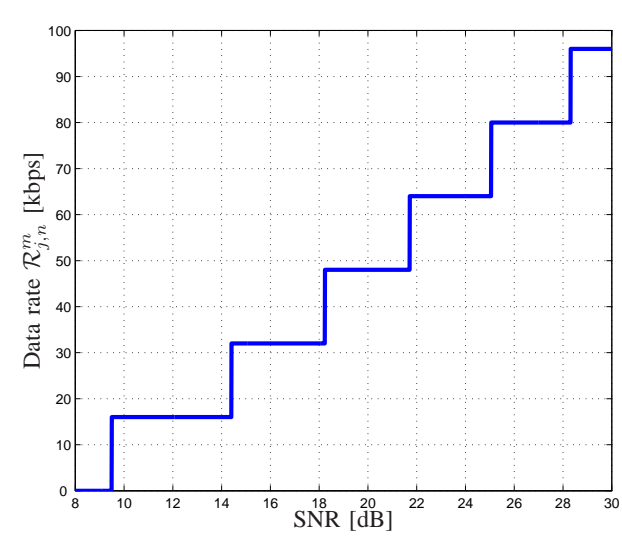

Fig. 1. Link adaptation curve of the subcarrier.

\section{Algorithm Description}

In order to compare with the performance of the proposed solution, classical RRA algorithms were used in this work. The classical algorithms are: Rate Maximization and Rate Adaptive. The description of those algorithms is presented in the following, together with a description of the proposed solution using GA. In order to evaluate only the subcarrier assignment schemes, the transmission power is assumed equal in all subcarriers in all solutions presented in the following, although schemes that also involve power allocation is possible in the presented algorithms.

\section{A. Rate Maximization}

One of the first problems studied in OFDMA RRA was the RM. The objective of this algorithm is to maximize the sum of the UE data rates subject to the constraint that one subcarrier cannot be shared by more than one UE at the same time. The problem formulation is presented in equation (4).

$$
\max _{\mathbf{x}} \sum_{j} r_{j}(\mathbf{x}) .
$$

The solution to this problem is very simple. Each subcarrier has to be assigned to the UE that has the highest channel gain on it. In spite of its simple solution, the RM problem is not suitable when one considers fairness issues. This algorithm can cause starvation of UEs at cell edge due to high path loss. This algorithm will be used to give the upper bound of total system data rate [1].

\section{B. Rate Adaptive}

In the RA approach, the objective is to maximize the lowest UE throughput. The problem formulation is presented in equation (5).

$$
\begin{array}{ll}
\max _{\mathbf{x}} & \epsilon \\
& \text { subject to } \\
& r_{j}(\mathbf{x}) \geq \epsilon \quad \forall j .
\end{array}
$$


The RA problem belongs to the group of combinatorial programming ones that have no immediate solution. Rhee et al. proposed a sub-optimal solution to the RA problem in [3] and concluded that its performance is comparable to the optimal one. The solution provided in the paper will be used here for the purpose of comparisons in our simulations.

\section{Proposed Framework}

1) Problem Formulation: We formulate a problem where the objective is to maximize the number of UEs satisfied with their respective transmission rates, where each subcarrier is allocated to only one UE. If the rate requirement for the satisfaction of the UEs is $R_{r e q}$ and $r_{j}$ is the obtained transmission rate by the $\mathrm{UE} j$, this problem can be mathematically formulated as:

$$
\max _{\mathbf{x}} \sum_{\forall j} U\left(r_{j}(\mathbf{x})-R_{r e q}\right) .
$$

The optimization criterion or utility function of this formulation is composed by a sum of unit step functions $U\left(r_{j}-R_{r e q}\right)$ where each one refers to one UE and assumes the value 1 if the UE $j$ is satisfied with the obtained transmission rate $r_{j}$, that is $r_{j} \geqslant R_{r e q}$ or 0 , otherwise $\left(r_{j}<R_{r e q}\right)$. Therefore, this utility function implies on the maximization of the number of satisfied UEs. As the formulation (6) is a combinatorial problem, we use Genetic Algorithms (GAs) to solve it.

2) Modeling and Solution Using Genetic Algorithm: GAs are optimization algorithms inspired on the theory of natural selection. They combine the principle of survival of the fittest individual with randomized information exchange (reproduction) to exploit past information and speculate on new search points with expected improved performance. The main advantages of GAs in relation to traditional optimization methods (like calculus-based approaches) are its robustness, efficiency, and flexibility.

GAs operate on a set of elements called population. Each element, called individual or chromosome represents a possible solution. The chromosomes are codified according to the problem nature. In this case, a chromosome is an integer string whose length is the number of subcarriers $N$ to be assigned to $J$ UEs and each integer number in the string corresponds to a UE of the set $\{1,2,3, \ldots, J\}$. Fig. 2 illustrates this codification.

\begin{tabular}{|c|c|c|c|c|c|c|c|}
\hline $\begin{array}{c}\text { Subcarrier 1 } \\
\text { UE 4 }\end{array}$ & $\begin{array}{c}\text { Subcarrier 2 } \\
\text { UE 7 }\end{array}$ & $\begin{array}{c}\text { Subcarrier 3 } \\
\text { UE 1 }\end{array}$ & $\begin{array}{c}\text { Subcarrier 4 } \\
\text { UE } J\end{array}$ & $\ldots$ & Subcarrier $N-2$ & Subcarrier $N-1$ & Subcarrier $N$ \\
UE 7 & UE 2 & UE 4 \\
\hline
\end{tabular}

Fig. 2. Structure of the chromosome.

GAs use fitness functions in order to guide the optimization process. These fitness functions are closely related to the objective to be optimized. The higher the fitness of a chromosome, the higher the quality of the solution represented by it. In this problem, the following fitness function is used:

$$
\sum_{\forall j} U\left(r_{j}(\mathbf{x})-R_{r e q}\right)+\frac{R_{\text {total }}}{R_{\max }}
$$

The maximization of this fitness function is related to the maximization of the number of satisfied UEs with their transmission rates. The first term is the utility function in the formulation problem (6). The second term is the ratio between the sum of the transmission rates of all UEs $R_{\text {total }}$ and the maximum possible transmission rate $R_{\max }$ of the system (this occurs when all subcarriers are transmitting using the higher modulation order). Therefore, this term can assume values between 0 and 1 and consequently, this term has a magnitude smaller than the first term. Indeed, it will serve to rank solutions with the same number of satisfied UEs. That is, among solutions with the same number of satisfied UEs, those with higher total rate are preferable.

GAs start with a population of $q$ individuals and execute the genetic operators in each generation, producing successive populations. Four genetic operators are performed in each generation: selection, crossover, mutation and elitism. Selection is the process by which individuals are chosen according to their fitness in order to mate. Their probabilities of choice are proportional to their fitness function values, thus simulating natural selection. Crossover is the genetic process in which two selected members change genetic material. It consists of choosing a random cut point and dividing each of the two chromosomes into two parts. Two offsprings are then generated by concatenating the segment of one parent on the left of the cut point with the segment of the second parent on the right of the cut point. Fig. 3 illustrates this mechanism. This is the most important mechanism, through which new solutions are built from the best partial solutions. It is also possible to implement crossover where more than one cut point exist. Mutation consists in altering bits of the chromosomes randomly with a given probability (mutation rate). Fig. 4 illustrates this process, where the the sixth subcarrier was randomly selected to be mutated (UE 2 was randomly changed to UE 8). This operator has the effect of perturbing a certain chromosome in order to introduce new characteristics not present in any element of the parent population. Then, it becomes possible to escape from local minima. Elitism is the process in which the best individual of the population is maintained. Then, we avoid that the most adapted individual be lost in the next generation with the application of other operators.

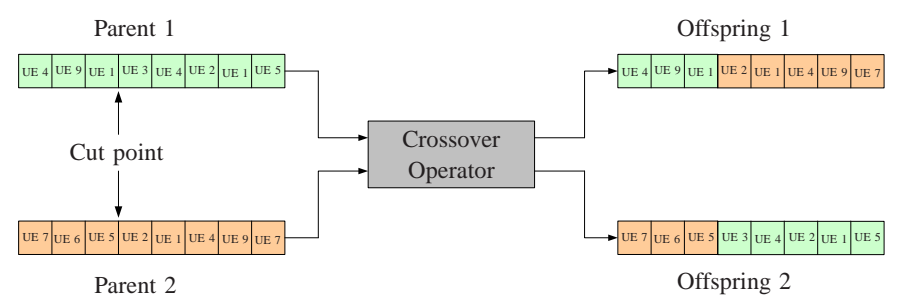

Fig. 3. Example of crossover.

Finally, the $q$ generated offsprings have their fitness evaluated. The new population is composed by the $(q-1)$ best adapted offsprings and the best individual from the current generation (elitism). This process is repeated in several generations, until the stop criteria (number of generations) is attained, as shown in Fig. 5. When the last generation 


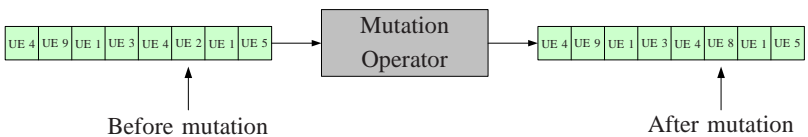

Fig. 4. Example of mutation.

finishes, the best individual of the final population will be the solution to the problem. Then, a sub-optimum solution, or even optimum, will be attained [5]. Indeed, an optimum solution is only guaranteed if the number of generations is high enough and the other algorithm parameters are well adjusted. Parameters related to GA used in this study are displayed in Table I.

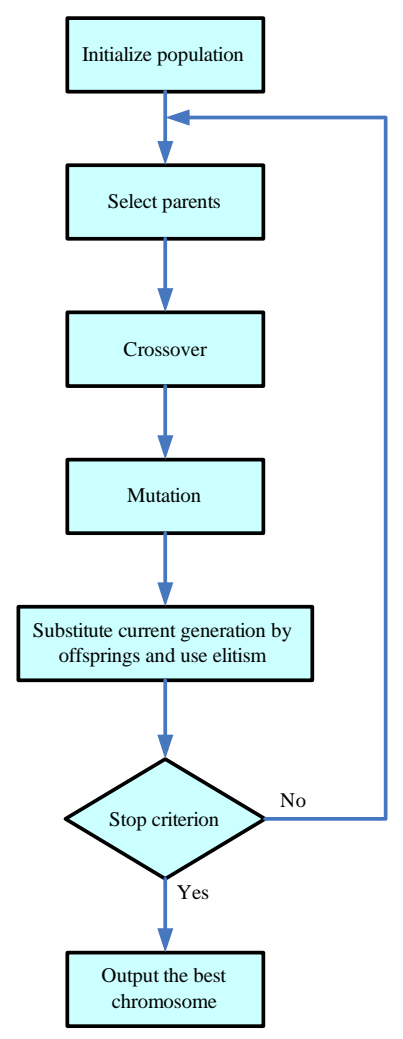

Fig. 5. Flowchart of GA.

TABLE I

Genetic Algorithm PARAmeters.

\begin{tabular}{c|c}
\hline \hline Parameter & Value \\
\hline \hline Number of generations & 800 \\
\hline Size of population & 200 \\
\hline Mutation Rate & $10 \%$ \\
\hline Kind of crossover & One cut point \\
\hline Use of elitism & Yes \\
\hline
\end{tabular}

\section{RESULTS}

In this section we present the numerical results obtained from computational simulations. In this paper we study the case of a single cell system. The multi-cell case is left for a forthcoming paper. We simulated 20 independent snapshots for several system loads and calculate the average number of satisfied UEs with their obtained transmission rates, as well as the average transmission rate of the system for each load for Rate Maximization, Rate Adaptive and the proposed solution using Genetic Algorithms. The main parameters used in the simulations are shown in the following.

The central operating frequency is $2 \mathrm{GHz}$. It is considered that there are 150 subcarriers available in the cell with a bandwidth of $15 \mathrm{kHz}$ for each subcarrier. Regarding propagation, the path loss $L[\mathrm{~dB}]$ at distance $d[\mathrm{~km}]$ is calculated by $L=128.1+37.6 \cdot \log _{10}(d)$. The shadowing standard deviation is $8 \mathrm{~dB}$. The modeled power budget has the following values. The noise power is $-123.24 \mathrm{dBm}$ per subcarrier, the total base station power is $20 \mathrm{~W}$, and the cell radius is $1 \mathrm{~km}$. Also, we assumed that the UEs are static and uniformly distributed in the cell coverage area. The transmission rate requirement $R_{r e q}$ which defines the threshold of satisfaction in this work is $256 \mathrm{kbps}$, for each UE.

In Fig. 6, we show the proportion of satisfied UEs with their obtained transmission rates for several simulated loads. We can see that the proposed scheme yields in a higher number of satisfied UEs with a larger performance gain in relation to the other schemes.

The proposed scheme performs better than RA algorithm because the first one tries to maximize the number satisfied UEs in a best-effort way according to fitness function (7), without worrying about the fairness in the distribution of the subcarriers, while RA algorithm takes into account in its formulation the fairness among the UEs, since it tries to maximize the minimum transmission rate among the UEs. Although one may think that a fair scheme is a good option, this procedure can be disastrous in some cases, because it can yield in situations where all the UE rates are balanced in a value lower than the rate requirement $R_{r e q}$ and, therefore, all links become unsatisfied while cause waste of resources. For that reason, a scheme that avoids this behavior, like the proposed algorithm, is more effective for the general case.

In relation to $\mathrm{RM}$, this algorithm does not take into account the UE rate requirements. Therefore, it is expected that concerning the percentage of the satisfied UEs, it presents the worst performance, as it can be seen in Fig. 6 where for the lower load in the figure (5 UEs) the proportion of satisfied UEs is only $39 \%$.

Other advantage of the proposed scheme is the fact that it prevents an abrupt decrease of the percentage of satisfied UEs in relation to the other algorithms. The relative decrease for GA is $11.9 \%$ when we vary the system load from 5 UEs to 20 UEs, while for RA and RM these decreases are $53.7 \%$ and $68.6 \%$, respectively. Therefore, the proposed scheme is more robust with the load variation.

In relation to the total cell transmission rate shown in Fig. 7, we can see that RM algorithm, as it was expected, outperforms the two other algorithms. This can be explained by the fact that this algorithm exploits the multi-user and frequency diversities, in such a way, that it schedules only the UEs with the best channel conditions in each subcarrier. Also note that the total cell transmission rate has a light increase with the system load due to the higher multi-user diversity. 


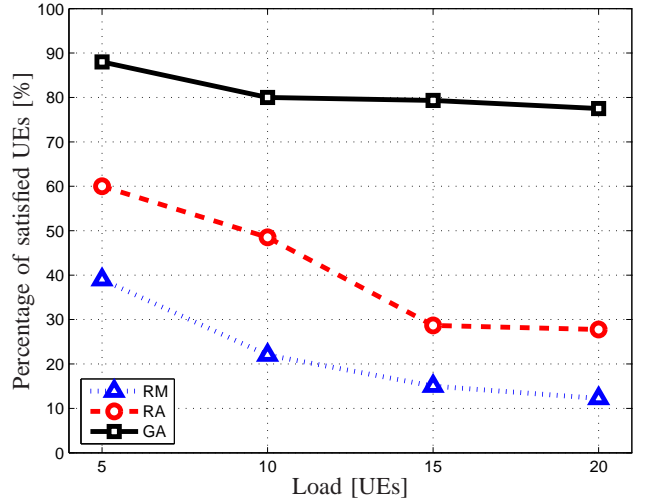

Fig. 6. Percentage of satisfied UEs for a rate requirement of $256 \mathrm{kbps}$.

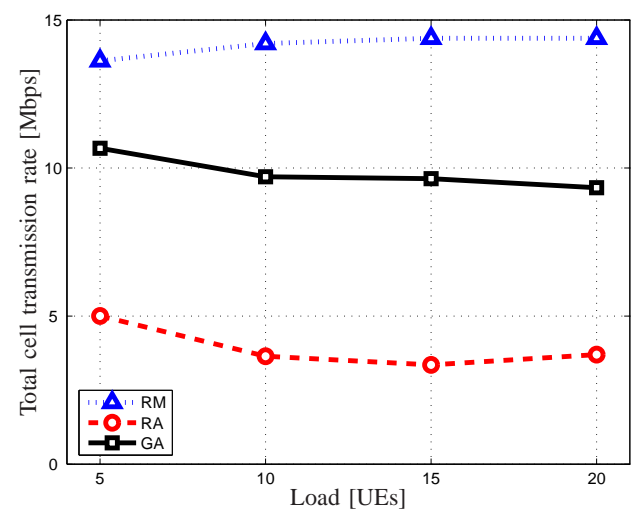

Fig. 7. Total cell transmission rate for a rate requirement of $256 \mathrm{kbps}$.

GA presents the second better performance on the total cell transmission rate, however, the difference on the total cell transmission rate between RM and GA is not so large, compared to the difference observed on the percentage of satisfied UEs. Therefore, we can consider that the proposed scheme is better in a general context.

\section{CONCLUSiON}

In this paper, we proposed a new Radio Resource Allocation problem in downlink of Orthogonal Frequency Division Multiple Access systems whose solution presents some advantages compared with other RRA problems such as Rate Adaptive and Rate Maximization. The solution of this new problem provides the maximization of the number of UEs with fulfilled rate requirements. In order to solve this problem, we utilized Genetic Algorithm by performing a suitable problem modeling.

The next steps are the evaluation of the solution of new problem in scenarios where the sessions have different rate requirements, and the conception of algorithms based on simple heuristics to solve the formulated problem in a smaller run time.

\section{REFERENCES}

[1] J. Gross and M. Bohge, "Dynamic Mechanisms in OFDM Wireless Systems: A Survey on Mathematical and System Engineering Contributions", Tech. Rep. TKN-06-001, Telecommunication Networks Group, Technische Universitat Berlin, May 2006.

[2] D. Kivanc, G. Li and H. Liu, "Computationally Efficient Bandwidth Allocation and Power Control for OFDMA", IEEE Journal on Selected Areas in Wireless Communications, Vol.2, pp. 1150-1158, November 2003

[3] W. Rhee and J. Ciof, "Increase in Capacity of Multiuser OFDM System Using Dynamic Subchannel Allocation", Proc. vehicular technology Conference (VTC), pp. 1085-1089, 2000.

[4] M. C. Sinclair, "Evolutionary Telecommunications: A Summary", Proc. GECCO'99 Workshop on Evolutionary Telecommunications: Past, Present and Future, pp. 209-212, 1999.

[5] S. M. Sait and H. Youssef, "Iterative Computer Algorithms with Applications in Engineering: Solving Combinatorial Optimization Problems", IEEE Computer Society Press, 1999.

[6] Y. Wang and F. Chen and G. Wei, "Adaptive Subcarrier and Bit Allocation for Multiuser OFDM System Based on Genetic Algorithm", Proceedings of International Conference on Communications, Circuits and Systems, pp. 242-246, 2005.

[7] I.C. Wong, Zukang Shen, B.L. Evans and J.G. Andrews, "A Low Complexity Algorithm for Proportional Resource Allocation in OFDMA Systems", IEEE Workshop on Signal Processing Systems, pp.1-6, Oct. 2004. 